\title{
Jam-free gear-clutch mechanism for load-sensitive step transmission in robotic joint
}

\author{
Maroay Phlernjai" (D) and Toshio Takayama
}

\begin{abstract}
This paper introduces a novel gear-clutch mechanism that takes advantage of the difference between the directions in the reaction forces that occur during meshing and jamming to ensure jam-free engagement of the gears. The proposed mechanism is a more compact advancement of the previously developed linear rack-tilting clutch used to provide a step change in the reduction ratio. Mathematical models of the jam-free and stable meshing condition of the proposed mechanism are developed and experimentally verified, along with a discussion and recommendations to be considered as design guidelines. Additionally, a singularly actuated robotic joint prototype is developed to examine the performance of the proposed clutch mechanism. The joint was driven by a small 2-W DC motor. The maximum output torque was $4 \mathrm{Nm}$ with a maximum travel range of over $200^{\circ}$ during the high-force phase, and the maximum speed was $252 \%$ with an infinite travel range during the high-speed phase. The mechanism exhibits potential for applications that benefit from a step transmission and long force-exerting travel range such as vices, grippers, and industrial punching and shearing machines, as well as robotic arms and power assist exoskeletons.
\end{abstract}

Keywords: Clutch mechanism, Jam-free, Transmission

\section{Background}

Many applications require changes in reduction ratiosfrom car transmission systems and industrial machines to robotic joints. For certain applications such as robot arms, having a fixed gear reduction ratio would limit the choice of speed and torque outputs. Given the same actuator, robot arms designed to lift heavy loads (high reduction ratio) would only be able to move substantially slower than the ones that were designed to handle lighter loads (low gear reduction), even when they operate without loads. Figure 1 illustrates a typical speed/torque graph use for gear sizing with DC motors. In order to deliver the desired output torque and speed, the motor and gear have to be chosen so that they can produce more torque/ speed than the target value. In the figure, operation point 1 refers to the target high speed and lower torque such

\footnotetext{
*Correspondence: phlernjai.m.aa@m.titech.ac.jp

The Department of Mechano-micro Engineering, Interdisciplinary

Graduate School of Science and Engineering, Tokyo Institute

of Technology, Yokohama, Japan
}

as when robot arm lifting light load. Operation point 2 refers to the target high output torque when robot arm lifting heavy object. If we size motor with a fixed reduction ratio gear, the output torque/speed line would have to cover both operation points and would result in motor with higher wattage when compared with the case where we can switch the reduction ratio and separately choose the torque/speed line to cover for each of the operation point. The ability to change the reduction ratio appropriately with respect to the load can provide a wider range of performance and allows the designer to use a smallersized motor, thus reducing the power consumption needed for the target application.

This need for switchable reduction ratios has led to the development of a continuously variable transmission (CVT). CVT drives have many configurations, such as those in [1-4]. Most CVT systems require a certain amount of time to achieve their final target ratio. A slow change may be unsuitable for certain applications that require quick movement, e.g., prosthetic devices and industrial machines. In this case, where the 


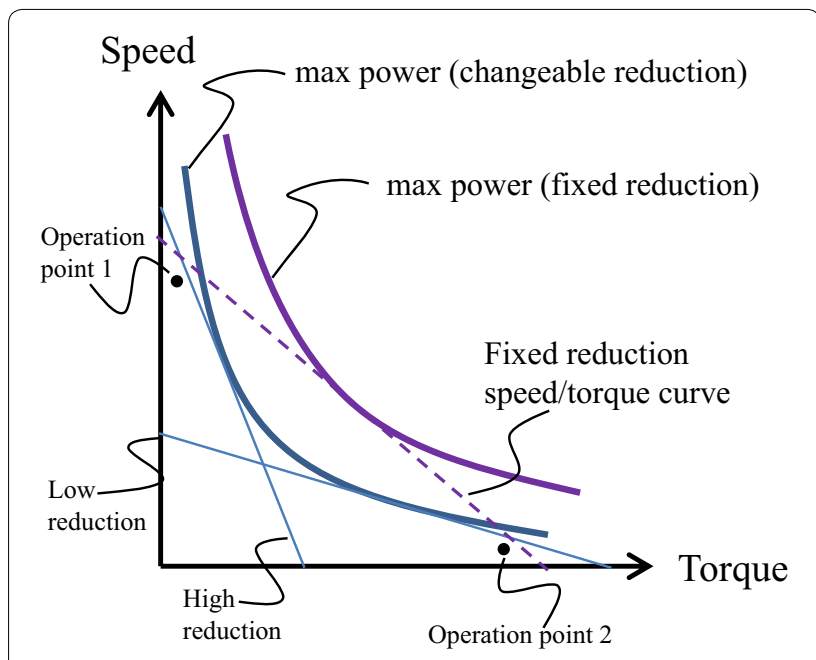

Fig. 1 Speed/torque graph for gear sizing

target application does not specifically require a continual change in reduction ratio, a step transmission is preferable.

Step transmission has been used in force magnification mechanisms developed in [5-7]. The mechanisms have the ability to swiftly shift from a high-speed (HS), low-force phase to a lower-speed, high-force (HF) phase. Although the developed mechanisms can solve the large time-consumption disadvantage of a CVT, the range of movement during the HF phase is limited and may not be suitable for applications such as robotic arms or exoskeletons that need to exert strong force over a long range of movement.

In our previous work on a dual-actuated robot hand [8], the hand provided a longer range of action in the HF phase by using a rack-gear clutch connection. To prevent gear teeth jamming during rack-gear engagement, a racktilting mechanism was introduced to the rack part, which will be discussed in the next section. The use of a rack gear increased the overall length of the mechanism, and design difficulties were encountered in our attempt to make the mechanism more compact. We also developed a more compact mechanism by replacing the dual-actuated mechanism with a single-actuated load-sensitive mechanism [9]. However, the use of a cable requires frequent maintenance and exhibits lower reliability than a gear-driven mechanism.

In this study, we propose a gear clutch mechanism that essentially changes the linear rack motion into a circular motion. Therefore, we can potentially produce a longer range of HF phase action with a relatively small mechanism size. Jam-free engagement is ensured by installing a link that can be tilted in the clutch mechanism. Conditions for jam-free engagements and their mathematical models are devised and experimentally confirmed in this paper. In addition, we also developed a robot joint prototype using the proposed gear clutch. The prototype is actuated by a small single DC motor and is experimentally verified to provide a step change in reduction ratio. The remainder of this paper is organized as follows: " Gear-clutch mechanism" describes the concept of the proposed gear-clutch, its model, and validation by experiments. "Robotic joint prtotype" discusses the developed robotic joint prototype using the proposed mechanism and its performance. Finally, "Conclusion" presents the conclusions of our study.

\section{Gear-clutch mechanism}

\section{Rack-tilting mechanism}

Figure 2 shows the simplified force magnification scheme of the rack-tilting mechanism previously developed in [8] (motors are not shown). After the finger is driven in HS phase by the motor $M 1$ to reach an object. Another motor $M 2$ then rotates the lead screw and drives the rack to the gear, providing HF phase of the hand. To ensure jam-free engagement, the rack pivot axis must be positioned in the sense that the reaction force from gear teeth jamming rotate the rack counter-clockwise, allow it to slip over the teeth and successfully engage the gear. Once engage, the teeth reaction force direction changes and keep the rack pressed against the support by clock-wise moment. The range of travel after engagement depends on the length of the rack. For applications that needs longer HF phase travel, the use of a rack and its travel length inevitably affect the overall length of the actuation unit, which may be too large to fit into the allocated space. Figure $3 \mathrm{com}$ pares the minimum allocation of linear rack and circular
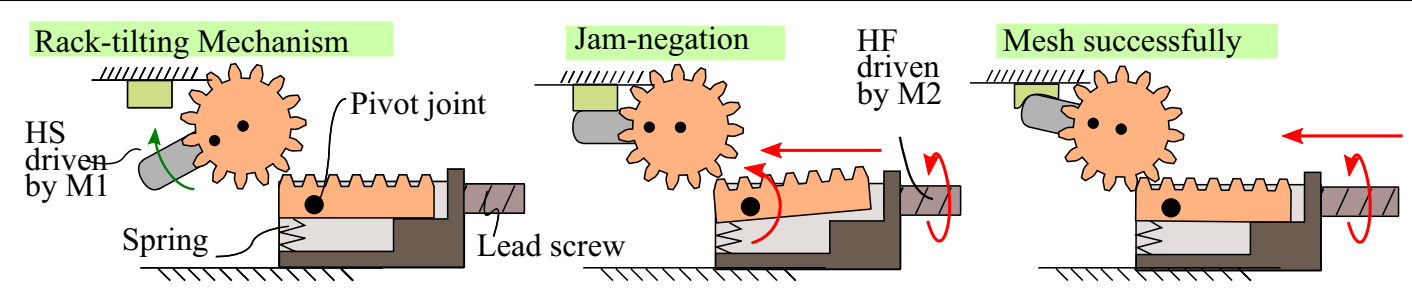

Fig. 2 Concept of the rack-tilting mechanism 

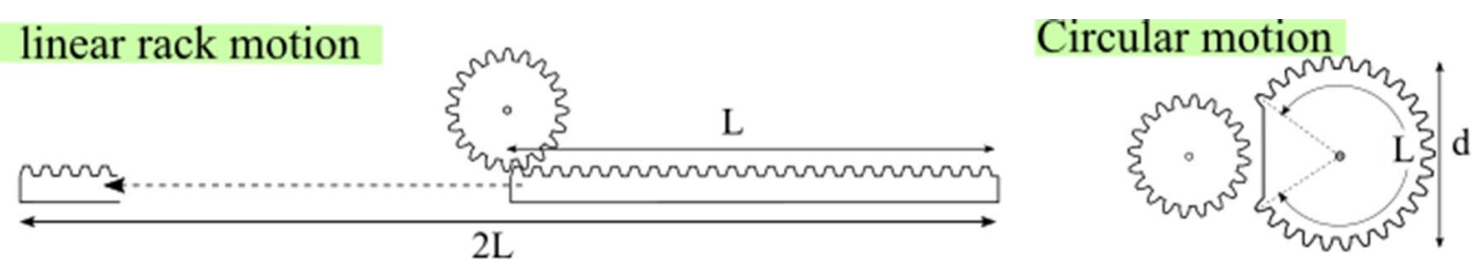

Fig. 3 Minimum allocation comparison of rack-tilting and the proposed gear clutch

gear clutch with rack gear length $L$. Even without considering allocation for lead screw and sliding support, the linear rack required allocation of at least $2 L$. Therefore, the circular design has a potential to greatly reduce the total length, leading to a more compact mechanism.

\section{Basic concept}

The basic concept of the proposed gear-clutch mechanism is similar to the rack-tilting mechanism-we use the difference in the direction of the reaction forces during jamming and meshing to allow jam-free engagement. Figure 4 shows the schematic of the gear-tilting clutch. Gear $G 1$ is driven after the $G 3$ movement has been stopped by the load object, thus driving $G 2$ into engagement. This requires a small tangential movement of the $G 2$ axis relative to the fixed-axis $G 1$ to ensure jam-free engagement (radial movement is not possible because excessive reduction in the G1-G2 center distance would cause the gears to stop rotating). Therefore, a tiltable link is introduced to connect $G 1$ and $G 2$, where $\theta$ is the angle between the G1-G2 and G2-G3 lines. The link can be tilted around G1 axis and will be referred to as "Tiltable link" in this paper. The spring is used to preload the link rotation and keep it pressed against the rotation limit. The rotation limit restrict clock-wise rotation of the link, prevent G2 and G3 center from being too close for engagement.

The proposed gear-clutch mechanism can be statically analyzed separately for cases of meshing and jamming situations.

\section{Stable meshing condition}

In the case of normal engagement or meshing, the gears transfer the input torque $\tau_{i n}$ to the output side by contacts between the gear teeth. For an involute teeth profile $[10,11]$, the normal force acting on the teeth has an angle equal to the pressure angle $\alpha$ of the gears. Figure 5 shows the free body diagram of each gear and the tiltable link in the mechanism for the meshing case. $F_{l t}$ and $F_{l r}$ is the tangential and radial component of the force acting on the link owing to G2, respectively. $r_{1}, r_{2}$, and $r_{3}$ are the pitch radii of the gears. The friction forces are neglected in order to simplify the model. Please note that for clarity, some forces, such as the reaction force at the fixed pivot joints, are not shown in Fig. 5. Only the forces related to the analysis are shown.

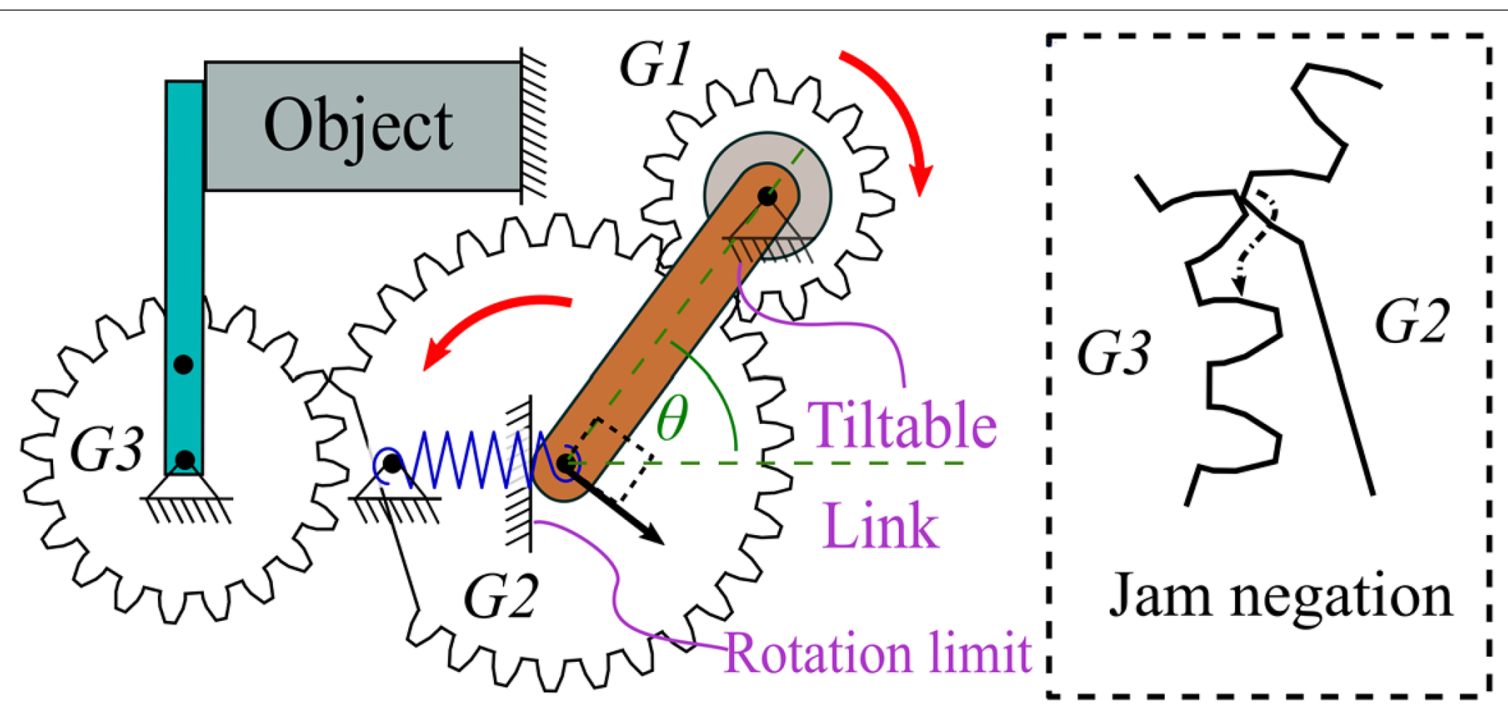

Fig. 4 Schematic of the proposed gear-clutch mechanism 


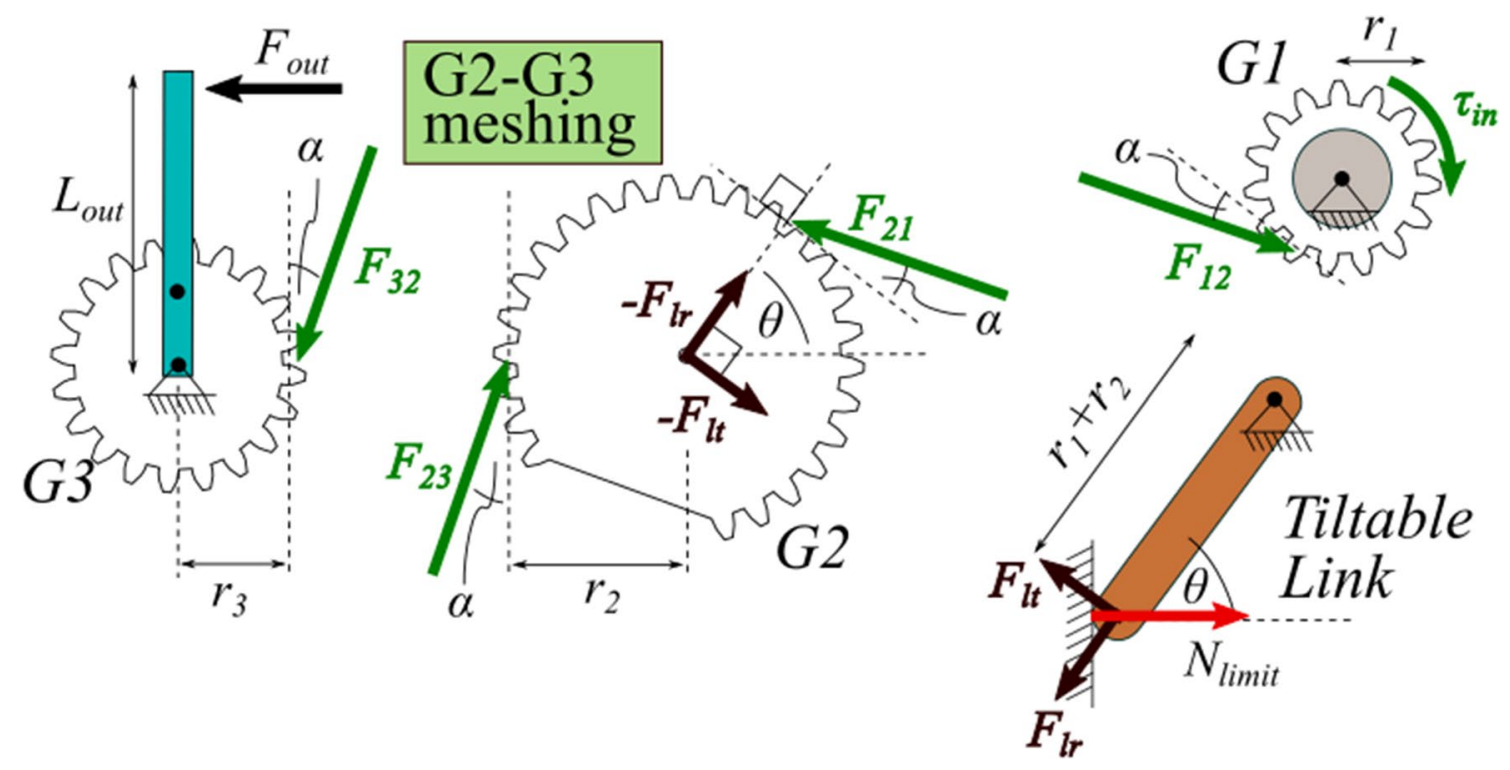

Fig. 5 Static analysis of the meshing condition

In static equilibrium, assuming frictionless contact,

$$
F_{l t}=\frac{\tau_{i n}(\cos \alpha+\cos (\theta+\alpha))}{r_{1} \cos \alpha}
$$

To maintain an appropriate center distance for the meshing position, $F_{l t}$ has to create a clockwise moment on the link and push it against the rotation limit. Therefore, in stable meshing, when $\tau_{\text {in }}>0$ and $\theta>0, F_{l t} \geq 0$. By combining these with Eq. 1, we obtain

$$
0<\theta \leq \pi-2 \alpha
$$

Equation 2 shows that the angle $\theta$ of the tiltable link connecting $G 1$ and $G 2$ must be within than a certain range or else $F_{l t}$ will reverse its direction and cause $G 2$ to disengage from the meshing position, which is not desirable. At $\theta=0$, the singular point of the link mechanism, a rotation of the link in any direction would cause the gears to always move away from each other, i.e. disengagement. Thus, such an unstable configuration is excluded from the allowable $\theta$ range.

\section{Jam-free condition}

Unlike the previous analysis on the meshing condition in which only the pressure angle $\alpha$ played a major role in determining the usable value of $\theta$, the force analysis in a jamming situation requires a closer look into the physical dimensions of the gears. Figure 6 shows the free body diagram of the gear-clutch mechanism during jamming. Please note that for clarity, some forces such as reaction forces at the fixed pivot joints are not shown. The reaction force pair $F_{23}, F_{32}$ points radially to the center of $G 3$ and acts at the jamming point at the intersection of the out-most circles of both gears. In this study, G2 is driven in the counter-clockwise direction for $\tau_{\text {in }}>0$. Therefore, only the upper intersection point is of concern. For the widely used involute gears, the jamming angle $\beta$ can be calculated from

$$
\beta=\cos ^{-1}\left(\frac{r_{3}\left(r_{3}+m\right)+r_{2}\left(r_{3}-m\right)}{\left(r_{3}+m\right)\left(r_{2}+r_{3}\right)}\right)
$$

where $m$ is the module of the gear teeth. Static equilibrium can be analyzed differently from the meshing case, assuming frictionless contact:

$$
F_{l t}=\tau_{i n}\left(\frac{1}{r_{1}}-\frac{r_{2} \sin (\theta-\beta)}{r_{1}\left(r_{2}+r_{3}\right) \sin \beta}\right)
$$

As we want the link to tilt a little and allow G2 to slip past the jammed tip, $F_{l t}$ must provide a counter-clockwise moment to the link and the link will then have a preloaded spring force $F_{s p}$ acting on it. In order to successfully negate the jamming, when $\tau_{\text {in }}>0, \theta>0$; on combining this with Eq. 4, we obtain

$$
\tau_{\text {in }}\left(\frac{1}{r_{1}}-\frac{r_{2} \sin (\theta-\beta)}{r_{1}\left(r_{2}+r_{3}\right) \sin \beta}\right)<-F_{s p} \sin \theta
$$

To simplify the problem, we consider the case when the spring preload is considerably weak compared to the link tangential force $F_{s p} \ll F_{l t}$ which is especially true for the intended use of the gear-clutch in step transmission where high input torque is supplied to G1. This simplify the calculation and we arrive at the condition for jam-free engagement 


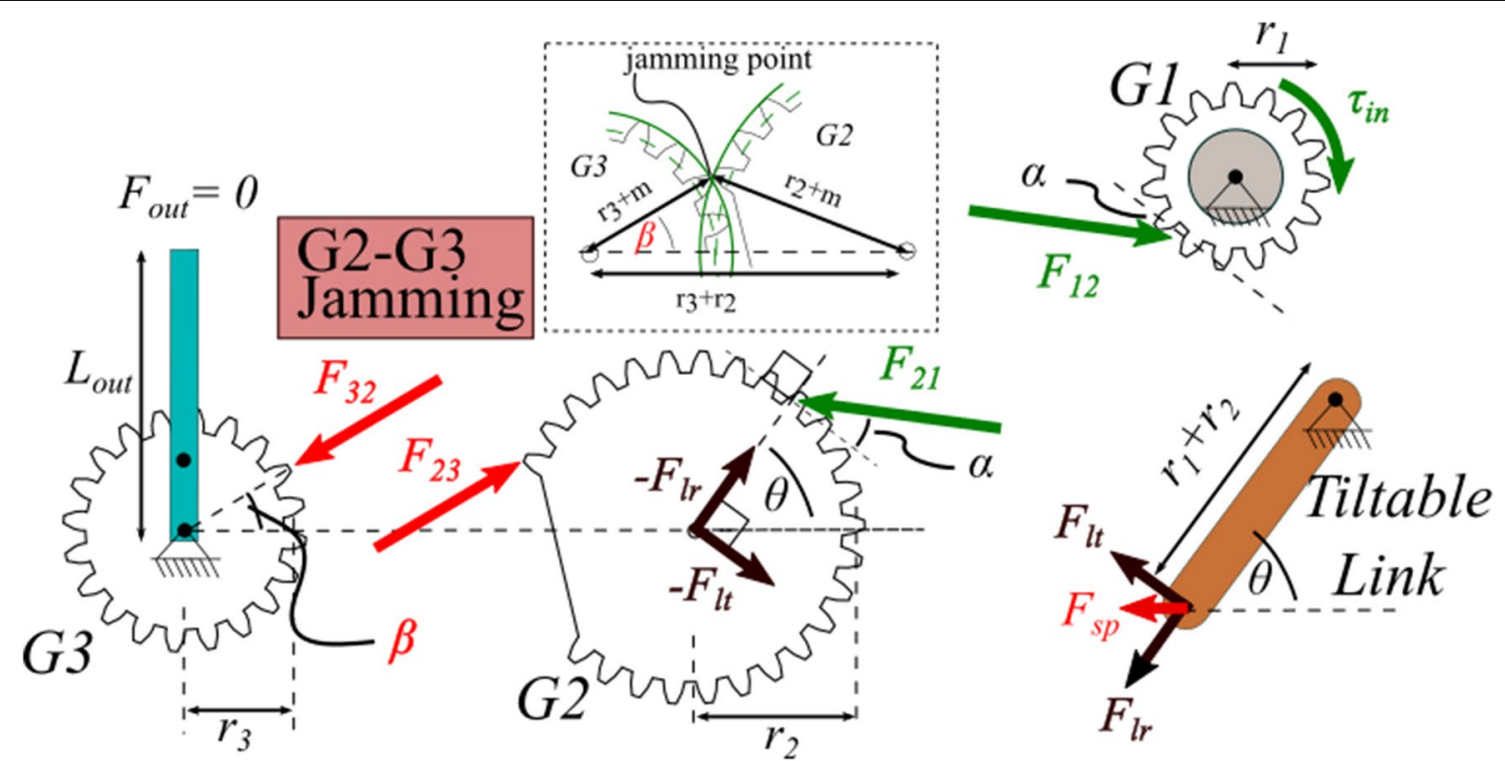

Fig. 6 Static analysis of the jamming condition

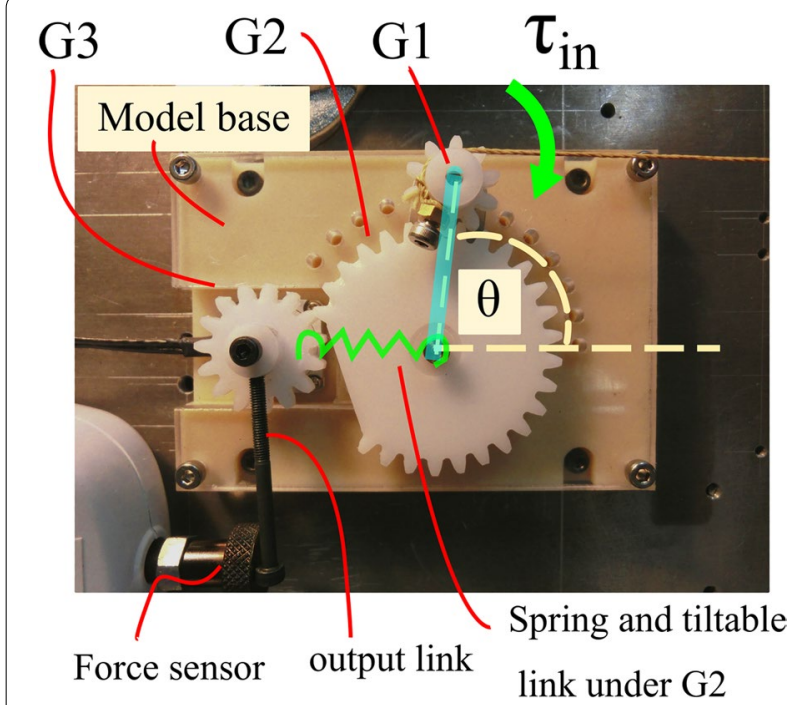

Fig. 7 Experiment setup for model validation of the gear-clutch mechanism

$$
\begin{aligned}
\frac{\pi}{2} & +\beta-\cos ^{-1}\left(\frac{\left(r_{1}+r_{3}\right) \sin \beta}{r_{2}}\right)<\theta<\frac{\pi}{2} \\
& +\beta+\cos ^{-1}\left(\frac{\left(r_{2}+r_{3}\right) \sin \beta}{r_{2}}\right)
\end{aligned}
$$

In order to design a working gear-clutch mechanism, the link angle $\theta$ must satisfy both the jam-free condition and the stable meshing condition, which can be derived from physical parameters of the gears.
Table 1 Specification of the experimental model

\begin{tabular}{lll}
\hline Parameter & Variable & Value \\
\hline Pitch radius of G1, G2, G3 & $r_{1}, r_{2}, r_{3}$ & $7.5,22.5,11.25 \mathrm{~mm}$ \\
Gears module & $m$ & $1.5 \mathrm{~mm}$ \\
Pressure angle & $\alpha$ & $20^{\circ}$ \\
Spring constant & $k$ & $0.052 \mathrm{~N} / \mathrm{mm}$ \\
Spring preloaded force & $F_{s p}$ & $0.312 \mathrm{~N}$ \\
Experiment input torque & $\tau_{\text {in }}$ & $25,50,75,100 \mathrm{mNm}$ \\
Experiment link angle & $\theta_{\exp }$ & $0^{\circ}-144^{\circ} / 12^{\circ} \mathrm{step}$ increment \\
Jam-free condition & $\theta$ & $86.29^{\circ}<\theta<158.76^{\circ}$ \\
Stable mesh condition & $\theta$ & $0^{\circ}<\theta \leq 140^{\circ}$ \\
\hline
\end{tabular}

\section{Model validation and discussion}

An experimental model for the gear-clutch mechanism is developed and used to validate the developed mathematical model of the jam-free and stable mesh conditions. Figure 7 and Table 1 show the experiment setting and its specifications, respectively. The three gears and the link are positioned as shown. Input torque $\tau_{i n}$ is generated by the cable tied to the $G 1$ hub. The rotation axis of $G 2$ is fixed on the link and is pulled by a weak spring placed below $G 2$ and G3. The output link is connected to G3. The model base is made of ABS plastic and polycarbonate. The gears are made of polyacetal. The tiltable link is made of aluminum.

First, we set the G3 position such that the G2-G3 jamming would always occur. The output link is positioned to barely touch the force sensor with zero readings. Then we used water weights to supply the input torque to $G 1$ via the cable. 


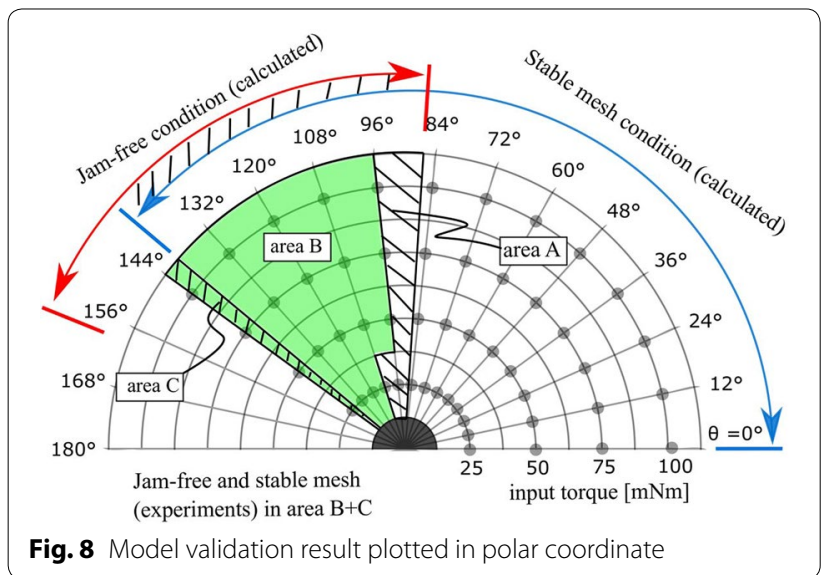

In the case of jamming, the reaction force at the teeth is directed radially to the center of G3 and cannot transmit torque. Therefore, we used the non-zero reading of the force sensor I (AIKOH, RX-50) as an indicator that G2 successfully negates the jamming and meshes properly with $G 3$.

We tested both the jam-free and mesh stability for different link angles $\theta_{\exp }$ and $\tau_{\text {in }}$ listed in Table 1 . All the angle in the experiment are displayed in $\left[^{\circ}\right]$. The experiment was conducted three times for each case to confirm the result. Figure 8 shows the experiment result in polar coordinates between $\theta$ and $\tau_{i n}$. All the cases considered in the experiment are represented by small dots, and the cases that actually resulted in both jam-free and stable meshing are highlighted in the graph (areas B and C). The results show that the intersected range of $\theta$ from the model can reasonably predict the actual usable range of $\theta$. At $\theta=96^{\circ}$, the minimum input torque for jam-free engagement can be calculated from Eq. 5 to be $\tau_{\text {in }}>21.3 \mathrm{mNm}$. With deviation from friction loss, the test model was able to start its jamfree engagement at $\operatorname{cin}=50 \mathrm{mNm}\left(\right.$ at $96^{\circ}$ ). Deviations from the calculated values are shown in the areas $\mathrm{A}$ and $\mathrm{C}$. The main cause for these deviations is the assumption of frictionless contact in the model. Friction impacts the jam-free condition negatively as shown in area A; it resists the slipping movement between the gear tips and makes it difficult to get out of the jamming position. On the other hand, in area $\mathrm{C}$, friction helps prevent the gears from disengaging even after the calculated stable meshing range is exceeded. However, if the input torque is low, the gear surface friction will not be large enough and it is possible that the gears will disengage. Therefore, as a design guideline of the proposed mechanism, choosing a $\theta$ close to but not exceeding the left limit of the intersected range is recommended.

\section{Robotic joint prototype}

Using the proposed clutch mechanism, we have also developed a robot joint prototype to examine the application of the clutch mechanism. The joint design goal is to load-sensitively produce a step change in reduction ratio and provide a long HF travel range. Figure 9 illustrates the components in the 3D CAD model. Figure 10 shows the joint prototype and its working concept. The developed prototype uses a single actuator in a float configuration, which allows the motor itself to act as a differential unit. The motor case output transmits torque to the low reduction gear train $(\mathrm{L})$ and the motor shaft output transmits torque to the high reduction gear train $(\mathrm{H})$ and the gear-clutch once a torque threshold determined by the slip-type torque limiter (TLES1-816-30W) is exceeded. The output torque $\tau_{\text {out }}$ can be determined from

High-speed phase: $\tau_{m} \leq \tau_{t h}$

$$
\tau_{\text {out }}=\tau_{m} \eta_{L} G_{L}
$$

High-force phase: $\tau_{m}>\tau_{t h}$

$$
\tau_{\text {out }}=\tau_{m}\left(\eta_{H} G_{H}+\eta_{L} G_{L}\right)-\tau_{t h} \eta_{H} G_{H}
$$

where $\tau_{m}$ represents the motor output torque, and $\tau_{t h}$ represents the effective threshold torque acting at the motor owing to the slip torque limiter. $G_{L}$ and $\eta_{L}$ are the reduction ratio and transmission efficiency of the low reduction gear train, respectively. Likewise, $G_{H}$ and $\eta_{H}$ are the reduction ratio and transmission efficiency of the high reduction gear train, respectively. We also define a maximum step transmission ratio as the maximum possible change in the reduction ratio between $\mathrm{HS}$ and $\mathrm{HF}$.

With maximum efficiency, $\eta_{H}, \eta_{L}=1$ and $\tau_{m} \gg \tau_{t h}$ :

$$
R_{\text {step }}=\frac{\tau_{\text {out }, H F}}{\tau_{\text {out }, H S}}=\frac{G_{H}+G_{L}}{G_{L}}
$$

Table 2 shows the prototype specifications. $\eta_{L}$ and $\eta_{H}$ are approximated from gears specification and number of the gear stages.

Owing to the limitation of space, the tiltable link is positioned opposite to $G 1$ but still on the line connecting $G 1$ and $G 2$. As the link was only required to be tilted by a small angle for jam-negation, positioning the link in this manner or instead using a sliding slot for G2, tangent to the $G 1$ circumference should provide the same result. A DC adapter is used to supply the input voltage to the rotating motor unit. The prototype is made mainly of aluminum and weighs $650 \mathrm{~g}$. The motor was a $6-\mathrm{V}$, $2-\mathrm{W}$ geared DC motor (Pololu 1163) and the power supply used was Kikusui PMC 18-5 with a constant current limit.

\section{Step transmission experiment}

We attached an aluminum link to the output joint (205$\mathrm{mm}$ moment arm) and drove the motor for the full duty cycle $6 \mathrm{~V}$ PWM until it stalled; we then reversed the motor and turned the link back. The maximum current is limited by the power supply to less than $30 \%$ of its stall current to 


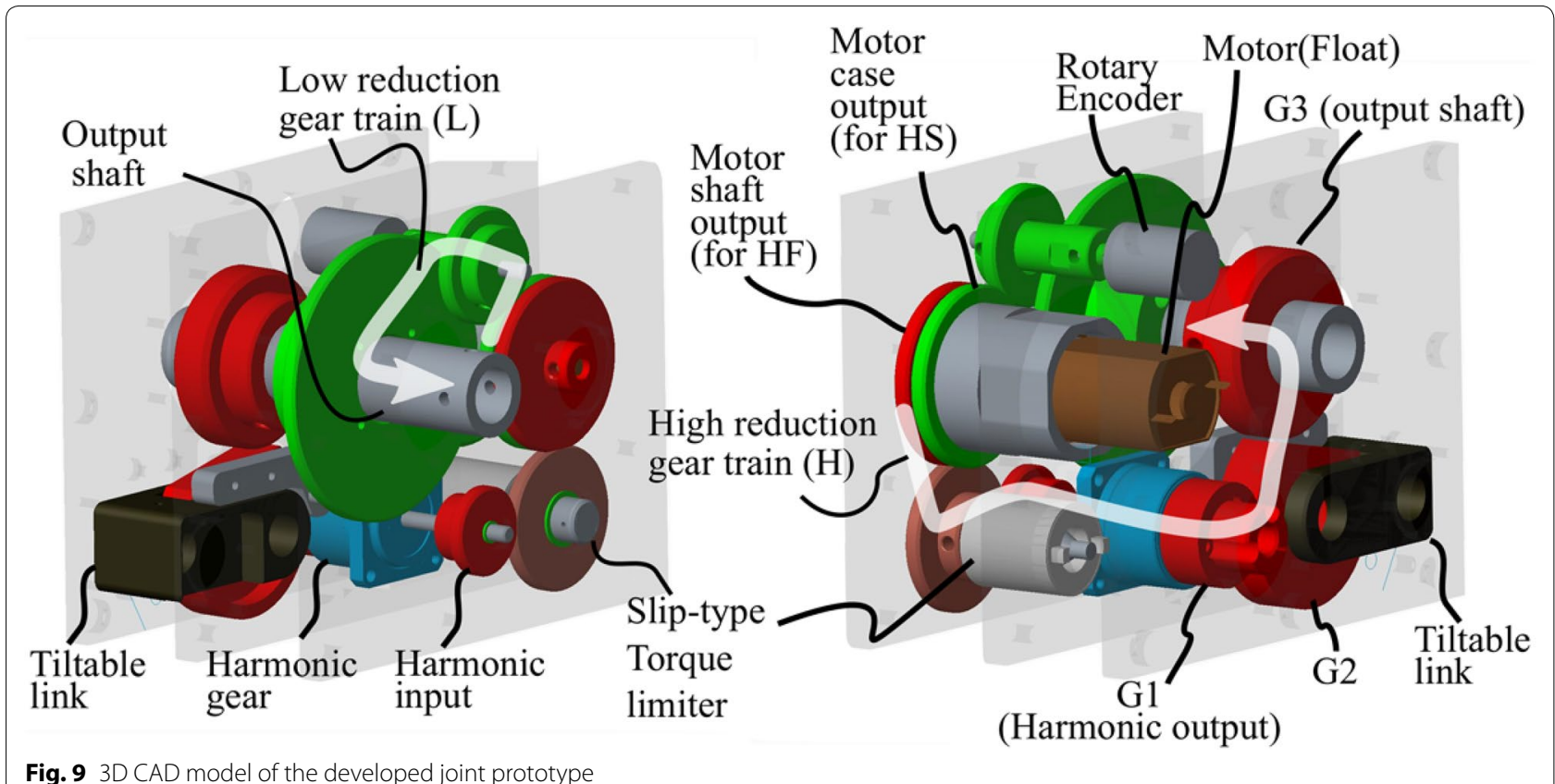

prevent overheating. Simultaneously, we measured output torque, output joint position, and average motor current at a sampling rate of $200 \mathrm{~Hz}$. Figure 11 shows the step transmission result. First, the link moved quickly in the HS phase and made contact with the force sensor. The output torque increased abruptly after the clutch engaged and the link entered the HF phase. In the return movement, the joint speed remained slow until the clutch disengaged; it then moved quickly in the HS phase again. The maximum joint speed in the HS phase is approximately $252^{\circ} / \mathrm{s}$, and the maximum torque in the HF phase is $6.57 \mathrm{Nm}$ with 1 -A motor current. The time delay in step transmission was in two parts, D and E. The delay D is the time used for the clutch to engage the gear. $E$ is the time used from engagement until the HF output is reached. In this case, D and E were approximately 0.43 s $0.3 \mathrm{~s}$ respectively. The jam-free engagement of the gear-clutch mechasnism in the joint prototype were also observed in additional file 1

\section{HF travel range experiment}

We also conducted another experiment to examine the prototype behavior and its maximum HF travel range when used to lift a load. An $80-\mathrm{mm}$ diameter pulley is connected to the output shaft, a $0.6-\mathrm{mm}$ diameter steel cable is fixed on the pulley at one end and connected to a water weights load at the other end. At first, the cable was loose. The motor was driven with the same current limit as the link experiment. The output pulley reeled the cable in the HS phase then continued to lift the water weight in the HF phase. After the maximum HF travel angle was reached, we reversed the voltage to lower the water weights down and loosen the tension in the cable. Figure 12 shows the experiment results. The HF travel angle is defined as shown. The maximum output torque was 4.8 Nm, which lifted the weight just above the ground and then stalled. At lower loads, the HF travel angles were in the range of $212^{\circ}-218^{\circ}$. The experiment with maximum $4 \mathrm{Nm}$ load can be viewed in Additional file 2 .

\section{Results and discussion}

The results show that the developed joint prototype could change its reduction ratio and produce an increased HF travel range according to the design. The maximum load of $4.8 \mathrm{Nm}$ was lower than the maximum torque in the step transmission experiment. This is probably because friction losses in the rotating gear train are higher than that in the case of the non-rotating one. In addition, cable swinging during the lifting might have increased the effective load and stalled the motor at a lower load value. The HF travel angles at the designed load of $4 \mathrm{Nm}$ and below were close to the designed maximum range of $220^{\circ}$. Deviations were caused by the variation in the output angle where the gear clutch starts engaging with the output gear. Therefore, it is safe to say that the prototype could provide an infinite travel range in $\mathrm{HS}$ with and upto a 4-Nm output torque over the range of $200^{\circ}$ in the HF phase. This $200^{\circ}$ travel range in the HF phase is long enough for applications like artificial elbow and knee joints. Time delay D occurs because the clutch has to fill the space between gear teeth of $G 2$ and $G 3$ in order to be 

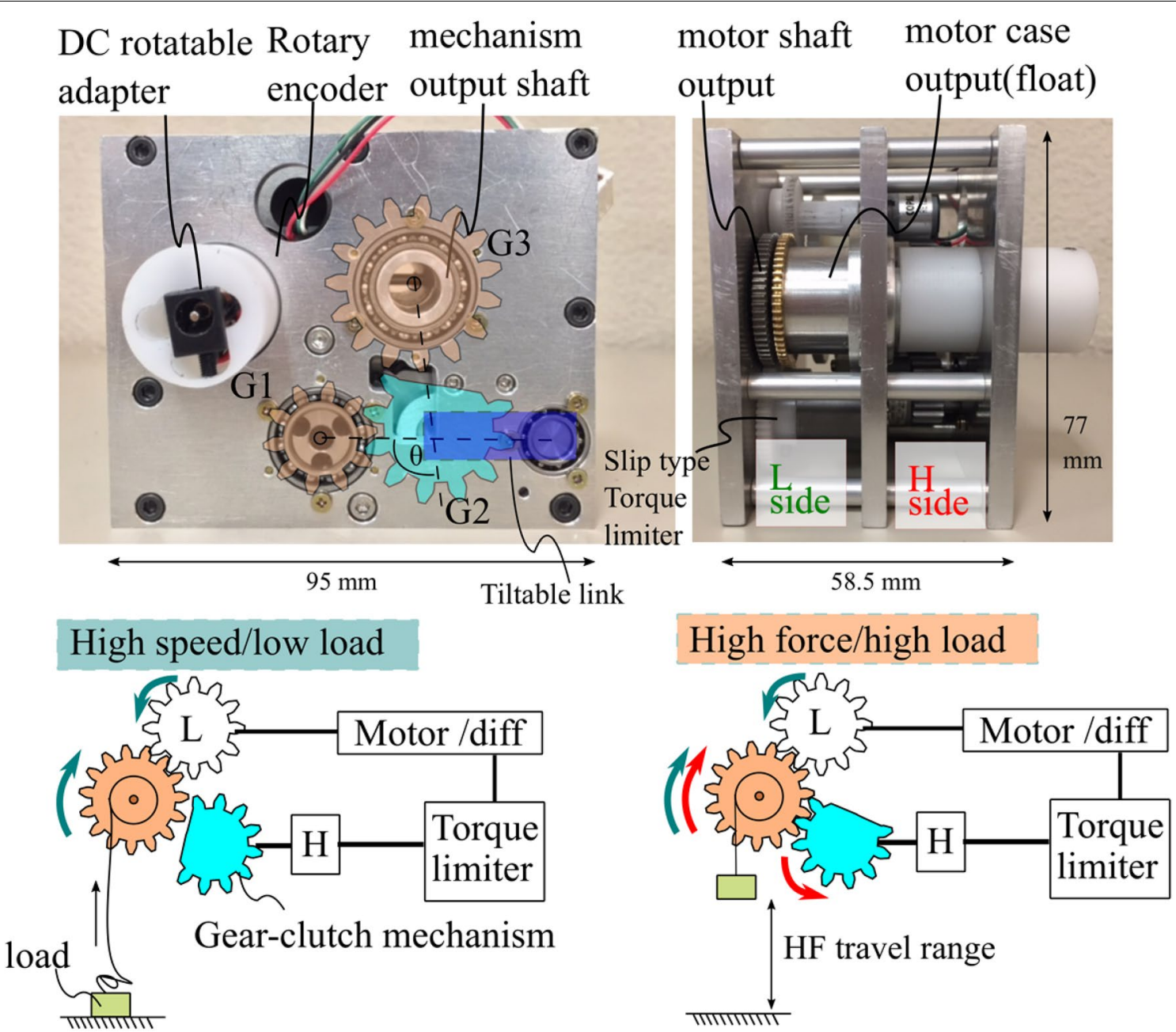

Fig. 10 Robotic joint prototype using the proposed gear-clutch mechanism

Table 2 Design specifications of the experimental model

\begin{tabular}{lll}
\hline Parameter & Variable & Value \\
\hline Designed maximum output torque & - & $4 \mathrm{Nm}$ \\
Maximum HF travel angle & - & $220^{\circ}$ \\
Approx. low reduction efficiency & $\eta_{L}$ & 0.90 \\
Approx. high reduction efficiency & $\eta_{H}$ & 0.57 \\
Low reduction ratio & $G_{L}$ & 4.44 \\
High reduction ratio & $G_{H}$ & 93.75 \\
Max step transmission ratio & $R_{\text {step }}$ & 22.09 \\
Link angle & $\theta$ & $98.5^{\circ}$ \\
Jam-free and stable mesh condition & $\theta$ & $64.47^{\circ}<\theta<140^{\circ}$
\end{tabular}

become fully engaged. D depends on the rotation speed of the clutch and module of the gear teeth. Therefore, it can be reduced by optimizing the mechanism design. On the other hand, delay E comes from the elasticity of the materials used in the mechanism and the load. As there is no perfectly stiff material, E could be lowered but could never be fully eliminated.

\section{Conclusion}

We developed a novel jam-free gear-clutch mechanism and its mathematical model for jam-negation and stable meshing conditions. The conditions were experimentally confirmed to reasonably predict the engagement behavior to a certain degree. We conclude that the small amount of deviation from the calculated conditions was mainly caused by friction forces, which were neglected in the model assumption. We also discussed the effects of friction and the actual design recommendations for the link angle $\theta$. As an example application, we developed a singleactuated robotic joint that can provide step transmission using the proposed gear-clutch. Its ability to abruptly change the reduction ratio and travel range in HF phase was verified through experiments. We can choose any two 


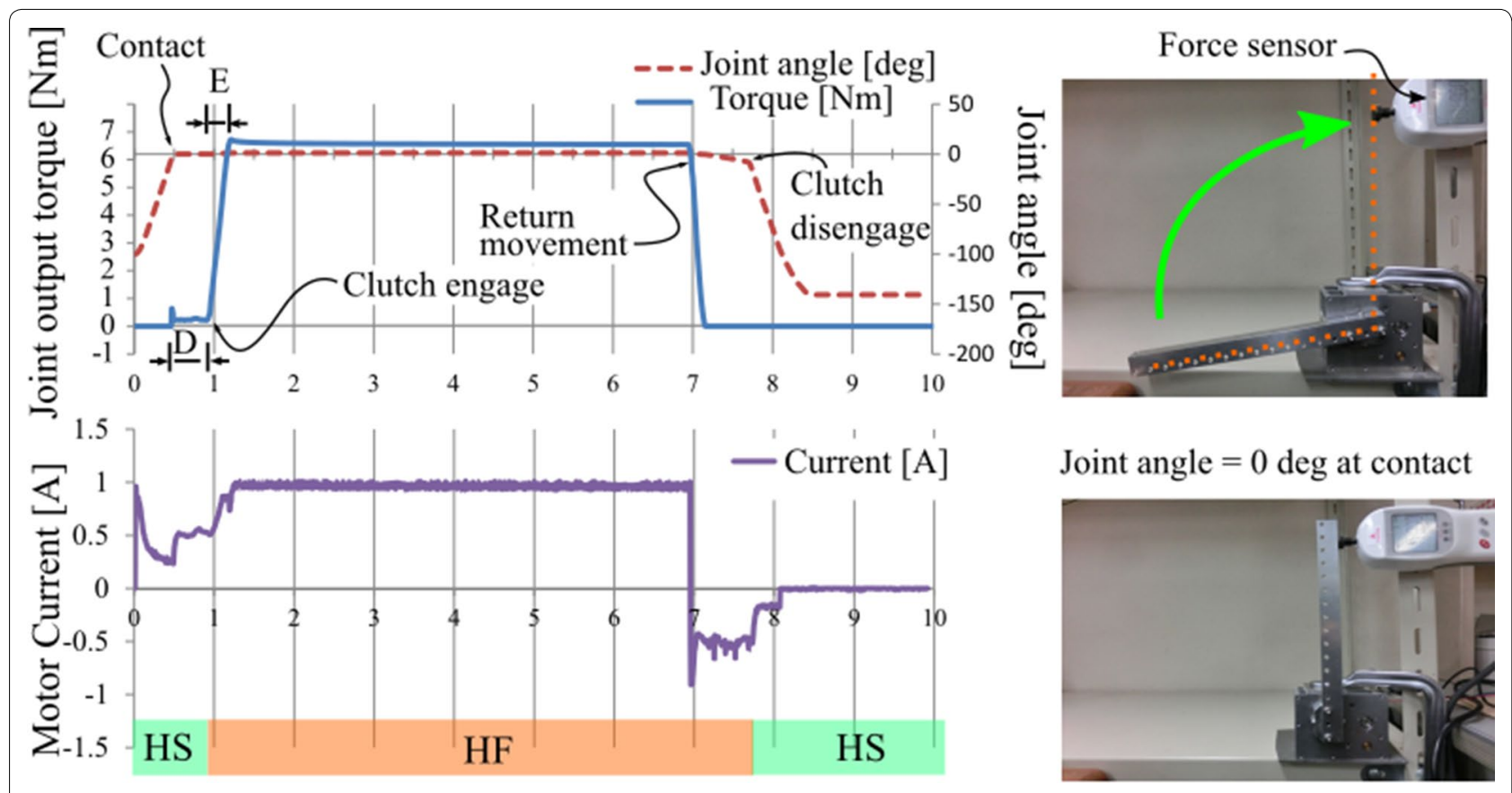

Fig. 11 Experiment results on step transmission of the prototype joint

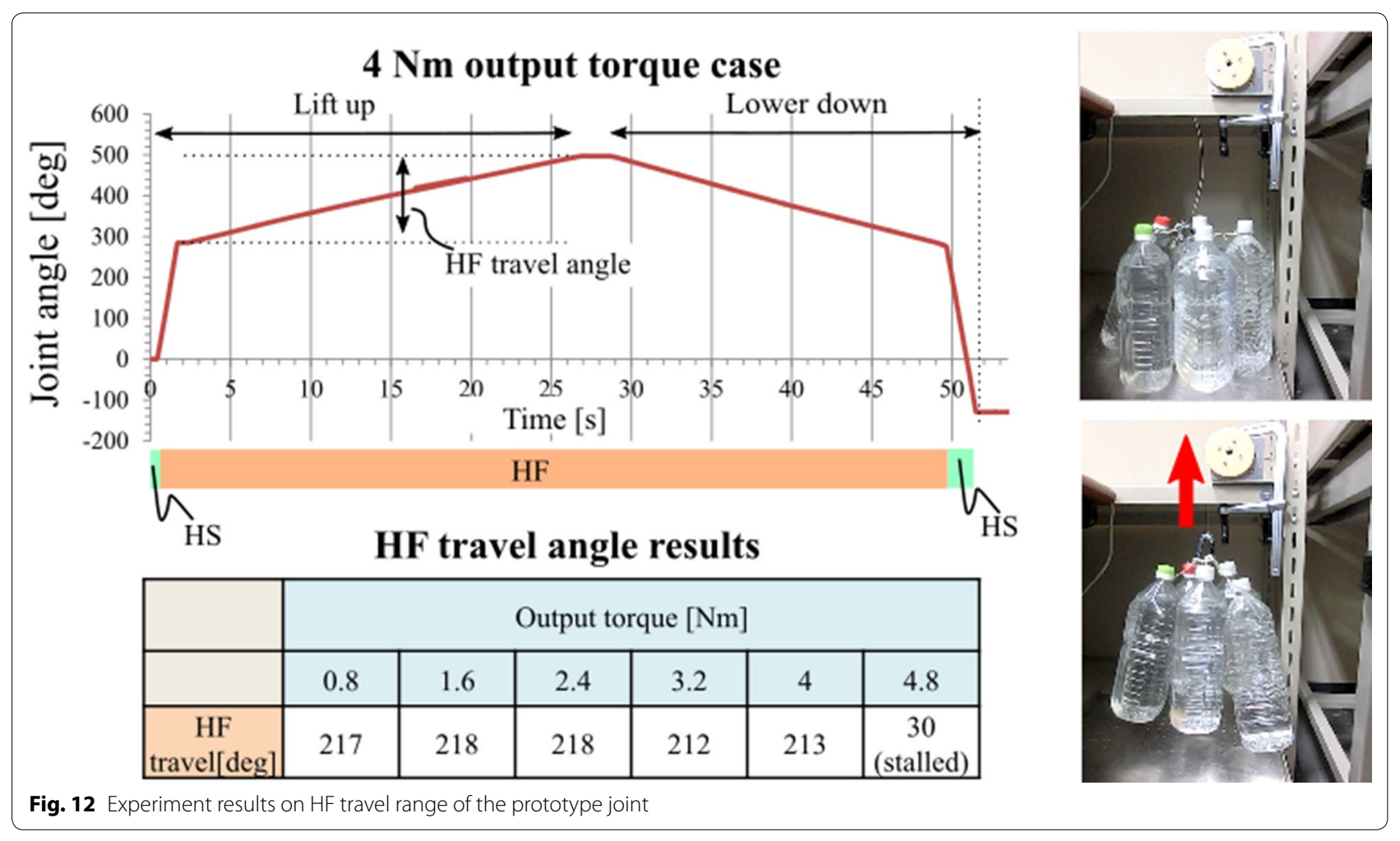


different reduction ratios ( $\mathrm{H}$ and $\mathrm{L}$ in Fig. 10) and design a gear-clutch mechanism with the required HF travel range to suit the target application. To our knowledge, the proposed mechanism shows a reliable engagement approach for applications that benefit from a step transmission and long travel range in the HF phase. Possible applications for this mechanism include robotic arms, power assist exoskeletons, and industrial machines such as vices, grippers, and punching and shearing machines.

\section{Additional files}

Additional file 1. Jam-free engagement of the gearclutch

Additional file 2. $\mathrm{HF}$ travel range experiment

\section{Abbreviations}

HS phase: high-speed phase; HF phase: high-force phase; L: low reduction gear train; $\mathrm{H}$ : high reduction gear train; $\mathrm{ABS}$ : acrylonitrile butadiene styrene.

\section{Authors' contributions}

MP carried out the conception and design, manufacturing, acquisition of data and drafting of the manuscript. TT carried out the conceptual supervising, data analysis and interpretation. Both authors read and approved the final manuscript.

\section{Authors' information}

Maroay Phlernjai received his B.E. degree from the Department of Mechanical Engineering, SIIT Thammasat University, Thailand in 2011. He received an M.E. degree from the Department of Mechano-micro Engineering, Tokyo Institute of Technology, Tokyo, Japan in 2014. Since 2014, he has been pursuing a doctoral degree in the Department of Mechano-micro Engineering, Tokyo Institute of Technology. His current research interests include mechanism design, robotic hands, force magnification systems, and prosthetic hands.

Toshio Takayama received his B.S., M.E., and Ph.D. degrees from the Department of Mechanical and Aerospace Engineering, Tokyo Institute of Technology, Tokyo, Japan, in 1998, 2000, and 2004, respectively. Since 2004, he has been an assistant professor in the Department of Mechano-Micro Engineering, Interdisciplinary Graduate School of Science and Engineering, Tokyo Institute of Technology, and he is currently an associate professor. His current research interests include the mechanical design of medical tools, robotic hands, and mobile robots.

\section{Competing interests}

The authors declare that they have no competing interests.

\section{Publisher's Note}

Springer Nature remains neutral with regard to jurisdictional claims in published maps and institutional affiliations.
Received: 16 February 2017 Accepted: 25 May 2017

Published online: 05 June 2017

\section{References}

1. Shin YJ, Lee HJ, Kim K-S, Kim S (2012) A robot finger design using a dual-mode twisting mechanism to achieve high-speed motion and large grasping force. IEEE Trans Robot 28(6):1398-1405. doi:10.1109/ TRO.2012.2206870

2. Spanjer SAJ, Balasubramanian R, Dollar AM, Herder JL (2012) Underactuated gripper that is able to convert from precision to power grasp by a variable transmission ratio. In: Proceedings of the ReMAR 2012 2nd ASME/IEEE International Conference on Reconfigurable Mechanisms and Robots. Springer, London, pp 669-679

3. Matsushita K, Shikanai S, Yokoi H (2009) Development of drum CVT for a wire-driven robot hand. In: IEEE/RSJ international conference on intelligent robots and systems, IROS 2009. pp 2251-2256. doi:10.1109/ IROS.2009.5354239

4. Tahara K, Iwasa S, Naba S, Yamamoto M (2011) High-backdrivable parallellink manipulator with continuously variable transmission. In: IEEE/RSJ international conference on intelligent robots and systems (IROS). pp 1843-1848. doi:10.1109/IROS.2011.6094423

5. Takayama T, Yamana T, Omata T (2012) Three-fingered eight-DOF hand that exerts 100-N grasping force with force-magnification drive. IEEE/ ASME Trans Mechatron 17(2):218-227. doi:10.1109/TMECH.2010.2094198

6. Takaki T, Omata T (2011) High-performance anthropomorphic robot hand with grasping-force-magnification mechanism. IEEE/ASME Trans Mechatron 16(3):583-591. doi:10.1109/TMECH.2010.2047866

7. Takaki T, Omata T (2007) Grasp force magnifying mechanism for parallel jaw grippers. In: IEEE international conference on robotics and automation. pp 199-204. doi:10.1109/ROBOT.2007.363787

8. Takayama T, Chiba G, Omata T (2013) Large grasp force hand with high compliance during high speed phase (in Japanese). Trans Jpn Soc Mech Eng Ser C 79(802):1893-1903

9. Phlernjai M, Takayama T, Omata T (2016) Passively switched cabledriven transmission for high-speed/high-force robot finger. Adv Robot 30(24):1559-1570. doi:10.1080/01691864.2016.1251336

10. Maitra GM (1994) Handbook of gear design. McGraw-Hill Professional, New York

11. Rao JS, Dukkipati RV (1992) Mechanism and machine theory. New Age International, New Delhi

\section{Submit your manuscript to a SpringerOpen ${ }^{\circ}$ journal and benefit from:}

- Convenient online submission

- Rigorous peer review

- Immediate publication on acceptance

- Open access: articles freely available online

- High visibility within the field

- Retaining the copyright to your article

Submit your next manuscript at springeropen.com 